\title{
Statistical Shape and Probability Prior Model for Automatic Prostate Segmentation
}

\author{
Soumya Ghose ${ }^{* \dagger}$, Arnau Oliver ${ }^{\dagger}$, Robert Martí ${ }^{\dagger}$, Xavier Lladó ${ }^{\dagger}$, Jordi Freixenet ${ }^{\dagger}$, Jhimli Mitra ${ }^{* \dagger}$, \\ Joan C. Vilanova ${ }^{\ddagger}$, Josep Comet $^{\S}$ and Fabrice Meriaudeau* \\ *Le2i-UMR CNRS 5158, Université de Bourgogne, Le Creusot, France \\ Email: \{soumya.ghose, jhimli.mitra, fabrice.meriaudeau\}@u-bourgogne.fr \\ $\dagger$ Computer Vision and Robotics Group, Universitat de Girona, Girona, Spain \\ Email: \{sghose, aoliver, marly, llado, jordif, jmitra\}@eia.udg.edu

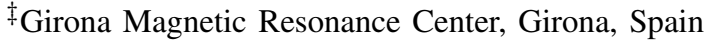 \\ $\S$ University Hospital Dr. Josep Trueta, Girona, Spain.
}

\begin{abstract}
Accurate prostate segmentation in Trans Rectal Ultra Sound (TRUS) images is an important step in different clinical applications. However, the development of computer aided automatic prostate segmentation in TRUS images is a challenging task due to low contrast, heterogeneous intensity distribution inside the prostate region, imaging artifacts like shadow, and speckle. Significant variations in prostate shape, size and contrast between the datasets pose further challenges to achieve an accurate segmentation. In this paper we propose to use graph cuts in a Bayesian framework for automatic initialization and propagate multiple mean parametric models derived from principal component analysis of shape and posterior probability information of the prostate region to segment the prostate. The proposed framework achieves a mean Dice similarity coefficient value of $0.974 \pm 0.006$, mean mean absolute distance value of $0.49 \pm 0.20 \mathrm{~mm}$ and mean Hausdorff distance of $1.24 \pm 0.56 \mathrm{~mm}$ when validated with 23 datasets in a leave-one-patient-out validation framework.
\end{abstract}

\section{INTRODUCTION}

Prostate cancer is the second most leading cause of death from cancer in American men [1]. Accurate prostate segmentation in TRUS may aid in biopsy therapy planning, motion monitoring, needle placement and multimodal image fusion between TRUS and magnetic resonance imaging (MRI) to improve malignant tissue collection during biopsy [2]. However, accurate computer aided prostate segmentation in TRUS images encounters considerable challenges due to low contrast of TRUS, heterogeneous intensity distribution and presence of micro-calcifications inside the prostate gland, speckle, and shadow artifacts. Moreover, inter patient prostate shape, size and deformation may vary significantly.

In the last decade, a number of semi-automatic prostate segmentation methods have been reported. These methods require expert intervention during initialization of the model or during refinement of the segmented contour. Often deformable models and statistical shape models are used to achieve segmentation in semi automatic methods like [3], [4]. However, it is necessary to use an automatic prostate segmentation method for TRUS guided procedures. Shen et al. [5] and Zhan et al. [6] presented an automatic method that incorporated a priori shape and texture information from
Gabor filters to achieve accurate prostate segmentation. However, the method is computationally expensive and therefore probably unsuitable for TRUS guided prostate intervention [2]. In recent years, Cosio et al. [7] reported an automatic method for prostate segmentation with active shape models [8]. However, the optimization framework of the genetic algorithm used is computationally intensive and unsuitable for TRUS guided intervention.

Cootes et al. [9] proposed an efficient framework to build a statistical model incorporating prior shape and texture information in their work of active appearance model (AAM). To address the challenges involved with prostate segmentation in TRUS images we propose a novel approach using multiple mean parametric models derived from principal component analysis (PCA) of prostate shape and posterior probabilistic values of the prostate region to segment the prostate in a multi-resolution framework. The performance of our method is compared with the traditional AAM [9] and also with our previous work [10]. In contrast, to the use of intensity and one mean model as in [9] and to the use of texture from Haar wavelet features of [10], posterior probabilistic information of the prostate region obtained in a Bayesian framework is used to train, initialize and propagate multiple statistical models of shape and texture. Statistically significant improvement is achieved with the use of multiple mean models when validated with 23 datasets, that have significant shape, size, and contrast variations of the prostate, in leave-one-patient-out validation framework. The key contributions of this work are:

- The use of iterative graph cut in identifying the prostate and use of the probability information of the prostate region obtained in a Bayesian framework in building the statistical model of texture.

- The use of the texture model in training, automatic initialization, propagation and selection of the optimum mean model.

\section{Proposed Segmentation Framework}

The proposed method is developed on the following major components: 1) the use of expectation maximization (EM) and Bayesian framework to determine the posterior probability of 


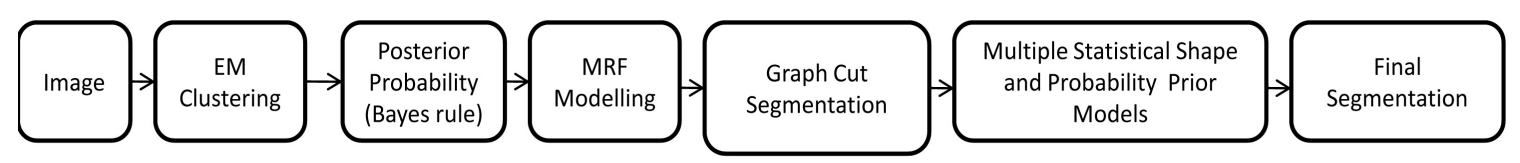

Fig. 1. Schematic representation of our approach. Abbreviations used EM $=$ Expectation Maximization, MRF $=$ Markov Random Field.

a pixel being prostate, 2) use of Markov random field (MRF) model to impose spatial constraints and improve the probabilistic representation of the prostate region, 3) estimation of the optimum label of the prostate region from graph cut segmentation on the MRF, 4) adapting AAM to incorporate the probabilities of the prostate region for training, initialization and propagation of the parametric model and 5) selection of one of the mean models depending on the error of fitting of the posterior probabilities to segment the prostate. The global schema of the method is given in Fig. 1.

\section{A. Bayesian Framework}

In traditional AAM [9], the point distribution model (PDM) [8] of the contour is aligned to a common reference frame by generalized Procrustes analysis [11]. Intensities are warped into correspondence using a piece wise affine warp and sampled from a shape-free reference to build the texture model. However, intensity distribution inside the prostate region may vary significantly from one dataset to another depending on the parameters of acquisition and nature of the prostate tissue of a patient introducing larger variabilities and hence producing an inaccurate texture model. Therefore, to reduce inter dataset intensity variabilities we propose to use the PCA of the posterior probabilities of the image pixels being prostate to build our texture model.

Firstly, K-means clustering is used to roughly cluster the pixels into two classes (prostate and non-prostate) from the intensities. The class means and standard deviations obtained from this rough clustering are then used as the initial estimates in an EM [12] based algorithm on Gaussian mixture model to determine the probability of a pixel being prostate from intensities. The E-step assigns the probabilities to the pixels depending on the current mean and standard deviation values of the classes, while in M-step the means and standard deviation values are re-estimated. Maximum a posteriori estimates of the class means and standard deviations are used to soft cluster the pixels. The likelihood of a pixel location in an image being prostate is obtained by normalizing the ground truth values of all the pixels for all the training images as

$$
P\left(x_{p s} \mid C_{p r s}\right)=\frac{1}{N} \sum_{i=1}^{N} G T_{i}
$$

where $P\left(x_{p s} \mid C_{p r s}\right)$ gives the probability of a pixel position being prostate with $x_{p s}$ being the pixel location (ps standing for position) and $C_{p r s}$ denoting pixels being prostate (prs stands for prostate). $G T_{i}$ represents the ground truth of the training image, $N$ being the total number of ground truth images. In our model, the class prior probability is estimated from the frequency of the pixels $(x)$ belonging to a class as

$$
P\left(C_{p r s}\right)=\frac{\sum_{i=1}^{p n m} x_{i}}{\sum_{j=1}^{m} x_{j}}
$$

where, $P\left(C_{p r s}\right)$ gives the class prior probability of being prostate, $x_{i}$ represents the pixels belonging to prostate region (total given by $p n m$ ) and $x_{j}$ represents the pixels in all training images (given by $m$ ). The probabilities of intensity (being prostate) obtained in the EM framework, location (being prostate) and class prior probability (prostate class) are used in a Bayesian framework to determine the posterior probability of a pixel being prostate. According to the Bayes rule,

$$
P\left(C_{i} \mid X\right)=\frac{P\left(X \mid C_{i}\right) P\left(C_{i}\right)}{P(X)}
$$

the posterior probability distribution $P\left(C_{i} \mid X\right)$ of a class is given by the prior $P\left(C_{i}\right)$ (i.e. $P\left(C_{p r s}\right)$ ) and the likelihood $P\left(X \mid C_{i}\right) . P(X)$ being equal for all classes can be removed from the formulation. Considering class conditional independence, the likelihood could be formalized as,

$$
P\left(X \mid C_{i}\right)=P\left(x_{p s} \mid C_{p r s}\right) \cdot P\left(x_{i n} \mid C_{p r s}\right)
$$

In equation (4) the likelihood $P\left(X \mid C_{i}\right)$ is obtained from the product of the probability of a pixel intensity being prostate $\left(P\left(x_{i n} \mid C_{p r s}\right)\right.$ ) obtained from EM framework ( $x_{i n}$ being pixel intensity) and the probability of a pixel location being prostate $\left(P\left(x_{p s} \mid C_{p r s}\right)\right)$ obtained from (1).

Further to impose spatial constraints over the pixel position we use MRF modeling over the posterior probabilities obtained from equation (3). MRF is a random process defined on a discrete lattice. For our case, the lattice is a $2 \mathrm{D}$ grid on the image plane. In 2D, we assume that $S=\{1,2, \ldots . N\} \times\{1,2, \ldots . N\}$ is the set on $N^{2}$ points called sites. For a fixed site $s$, a neighborhood $\mathbb{N}(s)$ is defined. Clique $c$ is defined as a set of sites, such that if $s_{i}, s_{j} \in c$ then $s_{j}$ is in the neighborhood of $s_{i}, s_{j} \in \mathbb{N}(i)$ and let $\mathbb{C}$ be the set of cliques. Assuming $X_{n}$ is a Markov chain we have,

$$
\begin{aligned}
& P\left(X_{n}=x_{n} \mid X_{k}=x_{k}, k \neq n\right)= \\
& P\left(X_{n}=x_{n} \mid X_{n-1}=x_{n-1}, X_{n+1}=x_{n+1}\right)
\end{aligned}
$$

i.e. the conditional distribution of $X_{n}$ depends only on its neighbors $X_{n-1}$ and $X_{n+1}$ in 2D. According to HammersleyClifford theorem [13], $X_{n}$ is a Markov field if and only if it follows a Gibb's distribution given by,

$$
P\left(X_{n}\right)=\frac{1}{Z} \exp \left[U\left(X_{n}\right) / T\right]
$$


where $Z=\sum \exp \left[U\left(X_{n}\right)\right]$ is a normalizing constant called partition function, $T$ is a constant called the temperature and the energy $U\left(X_{n}\right)=\sum V_{c}\left(X_{n}\right)_{c \in \mathbb{C}}$ is the sum of clique potentials $V_{c}$ over all possible cliques $c \in \mathbb{C}$ that depends on the local configuration on the clique $c$. The local potentials are determined using the Ising model [14].

According to the Ising model each particle can have one of two magnetic spin orientations +1 and -1 . Each particle interacts only with its neighbor and the contribution of each particle to the total energy of the system depends upon the orientation of its spin compared to its neighbor. Adjacent particles with same spin are in lower energy than those with dissimilar spin. To model our problem using the Ising model we assign initial labels +1 to the pixels with posterior probability greater than zero and assign -1 to other pixels. According to Ising model,

$$
P(x)=\frac{1}{Z} \exp \left[J \sum_{i \neq j} x_{i} x_{j}\right]
$$

where $x$ is a particular $N \times N$ configuration of $\pm 1, x_{i} \neq x_{j}$ are pair of neighboring pixels, $J$ represents the strength of the interaction between $x_{i}$ and $x_{j}$ that decrease the energy when both have same values and $Z$ is the partition function. Use of Ising prior in our models favors regions of coherence and imposes spatial restriction. We use the Gibb's sampling [14] to produce the Ising model.

The image now consists of pixels $z=\left(z_{1}, z_{2}, \ldots . z_{n}, \ldots z_{N}\right)$ with probabilistic values obtained with the Markov modeling. The segmentation of the image is expressed as an array of opacity values $\alpha=\alpha_{1}, \ldots \alpha_{N}$ at each pixel. In our case we use a hard segmentation to identify the prostate and hence $\alpha_{n} \in 0,1$ with 0 for background and 1 for the foreground i.e. the prostate. The parameters mean and covariance $(\theta)$ describe image foreground and background, represented with two Gaussian mixtures model (GMM). An energy function $E$ is defined so that its minimum produces good segmentation and is guided by foreground and background GMMs and that the opacity is coherent imposing spatial constraints. This is captured by a Gibbs energy given by,

$$
E(\alpha, k, \theta, z)=U(\alpha, k, \theta, z)+V(\alpha, z)
$$

depending on the GMM component variables $k$. The data term $U$ that represents the GMM is defined as,

$$
U(\alpha, k, \theta, z)=\sum_{n} G\left(\alpha_{n}, k_{n}, \theta, z_{n}\right)
$$

where $G$ is a Gaussian probability distribution. $V$, the smoothness term in equation (8) that maintains coherence in regions of similar probabilities and is given as,

$$
V(\alpha, z)=\gamma \sum_{(m, n) \in C}\left[\alpha_{n} \neq \alpha_{m}\right] \exp \left(-\beta \| z_{m}-\left.z_{n}\right|^{2}\right)
$$

where $[\phi]$ denotes the function taking values 0,1 for a predicate $\phi, C$ is the set of pairs of neighboring pixels and $\beta$ a constant that encourages smoothness in region of similar contrast. The energy $E$ defined in equation (8) is minimized in an iterative manner in three steps. Step 1 is done by simple enumeration of the $k_{n}$ values for each pixel. In step 2 Gaussian parameters the mean and covariance are estimated with expectation maximization algorithm in standard manner. Finally, in step 3 a global optimization is achieved using minimum cuts as done by Boykov and Jolly [17]. Iterative minimization from steps 1 to 3 of the total energy $E$ with respect to the three parameters $\alpha, \theta, k$ ensures convergence at least to a local minimum of $E$ [16]. The energy minimization assigns $\alpha$ value to each pixel where $\alpha=1$ corresponds to the foreground (the prostate) and $\alpha=0$ corresponds to background. However, the segmentation achieved does not incorporate any shape information of the prostate and hence produce some misclassified regions. To further improve the segmentation result we adapt AAM of Cootes et al. [9] to introduce probability values (obtained in the Bayesian framework) of the prostate region (obtained with iterative graph cut) to build our texture model and to impose shape restriction. AAM or statistical shape and texture model is discussed next with the adaptation of the model to introduce probability values of the prostate region in building the texture model.

\section{B. Statistical Shape and Texture Model (AAM)}

The process of building the parametric statistical model of shape and texture variations involves the task of building a shape model, a texture model, and a combined model of texture and shape and prior learning of the optimization space from the combined model perturbation. To build the shape model, a PDM is built by equal angle sampling of the prostate contours to determine the landmarks automatically. The PDM of the contours are aligned to a common reference frame by generalized Procrustes analysis. PCA of the aligned PDMs identifies the principal modes of shape variations. Posteriori probabilistic information (of pixels being prostate) of the segmented region are warped into correspondence using a piece wise affine warp and are sampled from a shape free reference similar to the AAM. PCA of the posterior probabilities of the segmented region obtained with Markov random field modeling and graph cuts algorithm is used to identify their principal modes of variation. The model may be formalized in the following manner. Let $s$ and $t$ represent the shape and posterior probability models, then

$$
s=\bar{s}+\Phi_{s} \theta_{s}, \quad t=\bar{t}+\Phi_{t} \theta_{t}
$$

where $\bar{s}$ and $\bar{t}$ denote the mean shape and posterior probability information respectively, then $\Phi_{s}$ and $\Phi_{t}$ contain the first $p$ eigenvectors (obtained from $98 \%$ of total variations) of the estimated joint dispersion matrix of shape and posterior probability information and $\theta$ represent the corresponding eigenvalues. The model of shape and posterior probability variations are combined in a linear framework as,

$$
b=\left[\begin{array}{c}
W \theta_{s} \\
\theta_{t}
\end{array}\right]=\left[\begin{array}{c}
W \Phi_{s}^{T}(s-\bar{s}) \\
\Phi_{t}^{T}(t-\bar{t})
\end{array}\right]
$$

where $W$ denotes a weight factor (determined as in AAM [9]) coupling the shape and the probability space. A third PCA of 
the combined model ensures the reduction in redundancy of the combined model, and is given as,

$$
b=V c
$$

where $V$ is the matrix of eigenvectors and $c$ the appearance parameters.

\section{Optimization and Segmentation of a New Instance}

In our model, we incorporate the traditional AAM optimization. The objective function of our model is similar to AAM. However, instead of minimizing the sum of squared difference of intensity between the mean model and target image, we minimize the sum of squared difference of the posterior probability of the mean model and the target image. The prior knowledge of the optimization space is acquired by perturbing the combined model with known model parameters and perturbing the pose parameters (translation, scale and rotation). A linear relationship between the perturbation of the combined model $(\delta c)$ and the residual posterior probability values $(\delta t)$ (obtained from the sum of squared difference between the posterior probability of the mean model and the target image), and between the perturbation of the pose parameters $(\delta p)$ and the residual posterior probability values are acquired in a multivariate regression framework as,

$$
\delta c=R_{c} \delta t, \quad \delta p=R_{p} \delta t
$$

where $R_{c}$ and $R_{p}$ refer to the correlation coefficients. Given a new instance, equation (14) is used as update parameters. Given a test image, the posterior probability values of the pixels being prostate is determined in the Bayesian framework section (II-A). The sum of the squared difference of the posterior probability values with the mean model is used to determine the residual value $\delta t$. The combined model $(\delta c)$ and the pose parameters $(\delta p)$ are then updated using equation (14) to generate a new shape and combined model and hence, new posterior probabilities. The process continues in an iterative manner until the difference with the target image remains unchanged.

\section{Multiple Mean Models}

The statistical shape and texture model assumes the shape and the texture spaces to be Gaussians. However, inter patient prostate shape and their intensities may vary significantly. In such circumstances, a single Gaussian mean model is inefficient to capture the variations of shape and texture spaces. To address this problem, we propose to use multiple Gaussian mean models. The scheme is as follows; initially the 1 st dataset is chosen as the reference to register datasets 3 to 23 to produce a mean model of shape and texture. This mean model is used to test dataset 2. The sum of squared difference of the posterior probabilities between the mean model and dataset 2 is recorded as the fitting error after the final segmentation. Likewise, with the fixed reference (dataset 1), we build the second mean model registering datasets 2 and 4-23 to test on dataset 3 and record the fitting error. The process is repeated for all datasets from 4-23. This provides 22 model fitting

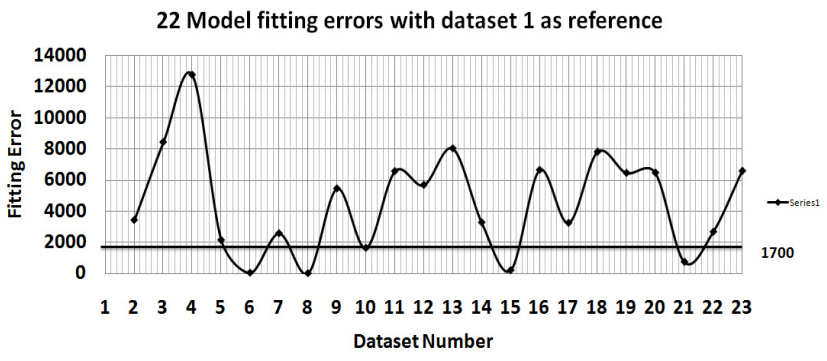

(a)

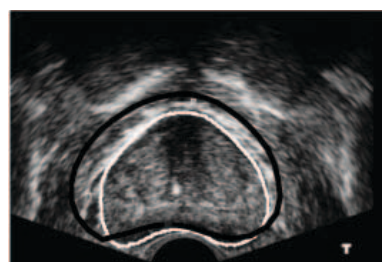

(b)

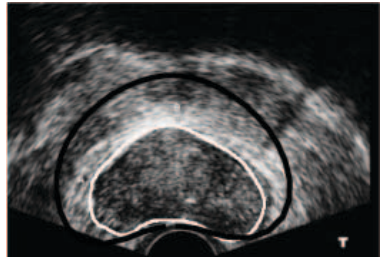

(d)

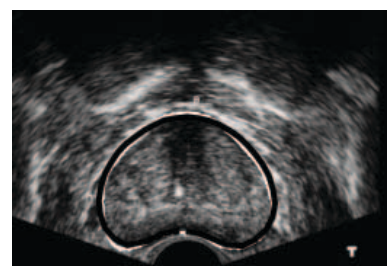

(c)

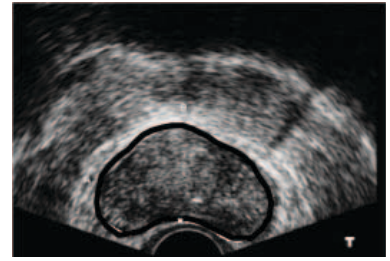

(e)
Fig. 2. (a) Mean models fitting errors for with dataset 1 as reference. (b), (d) Segmentation without multiple mean model, (c), (e) Segmentation with multiple mean model. The white contour gives the ground truth and the black contour gives the obtained result. Each row shows a different patient.

errors for the test datasets with dataset 1 as reference (Fig. 2(a)). Consequently, the reference dataset is changed from 2 through 23 and the entire process is repeated for all the datasets (23 in total). The entire procedure yields 23 graphs of model fitting errors (one for each dataset). We have analyzed these 23 model fitting error graphs and have observed that with less fitting error $(<2000$ units) we have higher accuracy in segmentation (in terms of different validation measures). This is not surprising considering the fact that the objective function of our optimization framework tries to minimize the fitting error between the mean model and the target image with respect to the pose parameters. Hence, an increase in fitting error indicates a reduction in segmentation accuracies. An empirical error value is determined from these graphs, above which, the segmentation accuracy is reduced (in our case the threshold value is 1700 units). The reference dataset that has a fitting error less than the empirical value for maximum number of test datasets is identified (dataset 1 in our case). The datasets below this fitting error are grouped together (datasets 1, 6, 8, 10, 15 and 21 (Fig. 2(a))) and are removed from further grouping. The process is repeated until all the datasets are grouped. These groups of datasets provide individual mean models (5 mean models in our case). However, increasing the 
TABLE I

Prostate segmentation Quantitative comparison (HD, MAD and MaxD in mm, Spec., Sens., And AcC., ARe For Specificity, Sensitivity AND ACCURACY RESPECTIVELY.)

\begin{tabular}{|l|l|l|l|l|l|l|l|}
\hline Method & DSC & HD & MAD & MaxD & Spec. & Sens. & Acc. \\
\hline AAM [9] & $0.92 \pm 0.04$ & $3.80 \pm 1.98$ & $1.26 \pm 0.76$ & $3.81 \pm 2.00$ & $0.91 \pm 0.04$ & $0.98 \pm 0.01$ & $0.97 \pm 0.05$ \\
\hline Ghose et al. [10] & $0.94 \pm 0.03$ & $2.59 \pm 1.21$ & $0.91 \pm 0.44$ & $2.64 \pm 1.19$ & $0.91 \pm 0.04$ & $0.98 \pm 0.01$ & $0.97 \pm 0.05$ \\
\hline B-AAM & $0.95 \pm 0.06$ & $2.53 \pm 1.94$ & $0.87 \pm 1.23$ & $2.35 \pm 2.10$ & $0.92 \pm 0.04$ & $0.97 \pm 0.04$ & $0.97 \pm 0.03$ \\
\hline Our Method & $0.97 \pm 0.01$ & $1.24 \pm 0.56$ & $0.49 \pm 0.20$ & $1.30 \pm 0.74$ & $0.96 \pm 0.01$ & $0.99 \pm 0.00$ & $0.98 \pm 0.00$ \\
\hline
\end{tabular}

TABLE II

QUALITATIVE COMPARISON OF PROSTATE SEGMENTATION

\begin{tabular}{|l|l|l|l|l|}
\hline Reference & Area Accuracy & Contour Accuracy & Datasets & Time \\
\hline Shen [5] & Error 3.98 $\pm 0.97 \%$ & Distance $3.2 \pm 0.87$ pixels & 8 images & $64 \mathrm{secs}$ \\
Ladak [4] & Accuracy $90.1 \pm 3.2 \%$ & MAD $4.4 \pm 1.8$ pixels & 22 images & - \\
Cosio [7] & - & MAD $1.65 \pm 0.67 \mathrm{~mm}$ & $11 \mathrm{minutes}$ \\
\hline Our Method & DSC $0.97 \pm 0.006$ & MAD $1.82 \pm 0.76$ pixels $/ 0.49 \pm 0.20 \mathrm{~mm}$ & 23 datasets $/ 46$ images & $22 \mathrm{~seconds}$ \\
\hline
\end{tabular}

number of mean models (decreasing the fitting error threshold) improves segmentation accuracy. In Fig. 2(b) and 2(d) we observe that segmentation error is high with one mean model in the Bayesian framework. However, segmentation accuracy improves with multiple mean models in the same framework (Fig. 2(c), 2(e)).

\section{EXPERIMENTAL RESULTS}

We have validated the accuracy and robustness of our method with 46 axial mid gland TRUS images of the prostate with a resolution of $348 \times 237$ pixels from 23 prostate datasets in a leave-one-patient-out evaluation strategy. During validation, a test dataset is removed and 5 mean models are built with the remaining 22 datasets. All the 5 mean models are applied to segment the test dataset. The mean model with the least fitting error is selected for accurate segmentation. The ground truth for the experiments are prepared in a schema similar to MICCAI prostate challenge 2009 [19], where manual segmentations performed by an expert radiologist are validated by an experienced urologist. We have used most of the popular prostate segmentation evaluation metrics like Dice similarity coefficient (DSC), 95\% Hausdorff Distance (HD) [19], mean absolute distance (MAD) [2], maximum distance (MaxD), specificity, sensitivity, and accuracy [3] to evaluate our method. Furthermore, the results are compared with the traditional AAM proposed by Cootes et al. [9], Ghose et al. [10] and to B-AAM (that uses posterior probability of the prostate region and a single mean model for segmentation).

It is observed from Table I that a probabilistic representation of the prostate texture in TRUS images and the use of multiple mean models significantly improves segmentation accuracy when compared to traditional AAM and to [10]. We used posterior probability information for automatic initialization and training of our statistical shape and texture model. As opposed to manual initialization of traditional AAM and [10], our model is initialized automatically. We achieved a statistically significant improvement in $t$-test $p$-value $<0.0001$ for DSC, HD and MAD compared to the other two approaches. A high DSC value and low values of contour error metrics of $\mathrm{HD}$ and MAD are all equally important in determining the segmentation accuracy of an algorithm. In this context, we obtained better segmentation accuracies compared to [9] and [10]. As observed in Table I, B-AAM (that uses posterior probability and a single mean model) produces better results compared to AAM justifying the use of posterior probability of the prostate region instead of intensity. However, our model which uses both posterior probability and multiple mean models, produces superior results compared to B-AAM, suggesting that the use of both posterior probability and multiple mean models are essential to improve segmentation accuracies. The improvement in segmentation accuracy with multiple mean model is evident from the last two rows in Table I.

A quantitative comparison of different prostate segmentation methodologies is difficult in the absence of a public dataset and standardized evaluation metrics. Nevertheless, to have an overall qualitative estimate of the functioning of our method, we have compared with some of the $2 \mathrm{D}$ segmentation works of the literature in Table II. Note that we may consider area overlap and area accuracy equivalent to that of DSC values, while average distance equivalent to that of average MAD. Analyzing the results we observe that our mean DSC value is comparable to area overlap accuracy values of Ladak et al. [4] and very close to the area overlap error of Shen et al. [5]. However, it is to be noted that we have used more images compared to Shen et al. Our MAD value is comparable to [5], [4], and [7]. From these observations we may conclude that qualitatively our method performs better in overlap and contour accuracy measures. Qualitative results of our method is illustrated in Fig. 3.

Our method is implemented in Matlab 7 on an Intel Core2Duo T5250, $1.5 \mathrm{GHz}$ processor and $2 \mathrm{~GB}$ RAM. The mean segmentation time of the method is $21.97 \pm 0.55$ seconds. Our mean segmentation time is better compared to Shen et al. [5] and Cosio et al. [7] with an un-optimized Matlab code. Due to off-line optimization of our statistical model of shape and probability prior the mean fitting time is 0.68 seconds which may be reduced further in an optimized $\mathrm{C}++$ or GPU environment. The process is computationally expensive in determining the posterior probability from MRF regularization. However, near real time implementation of MRF regularization 

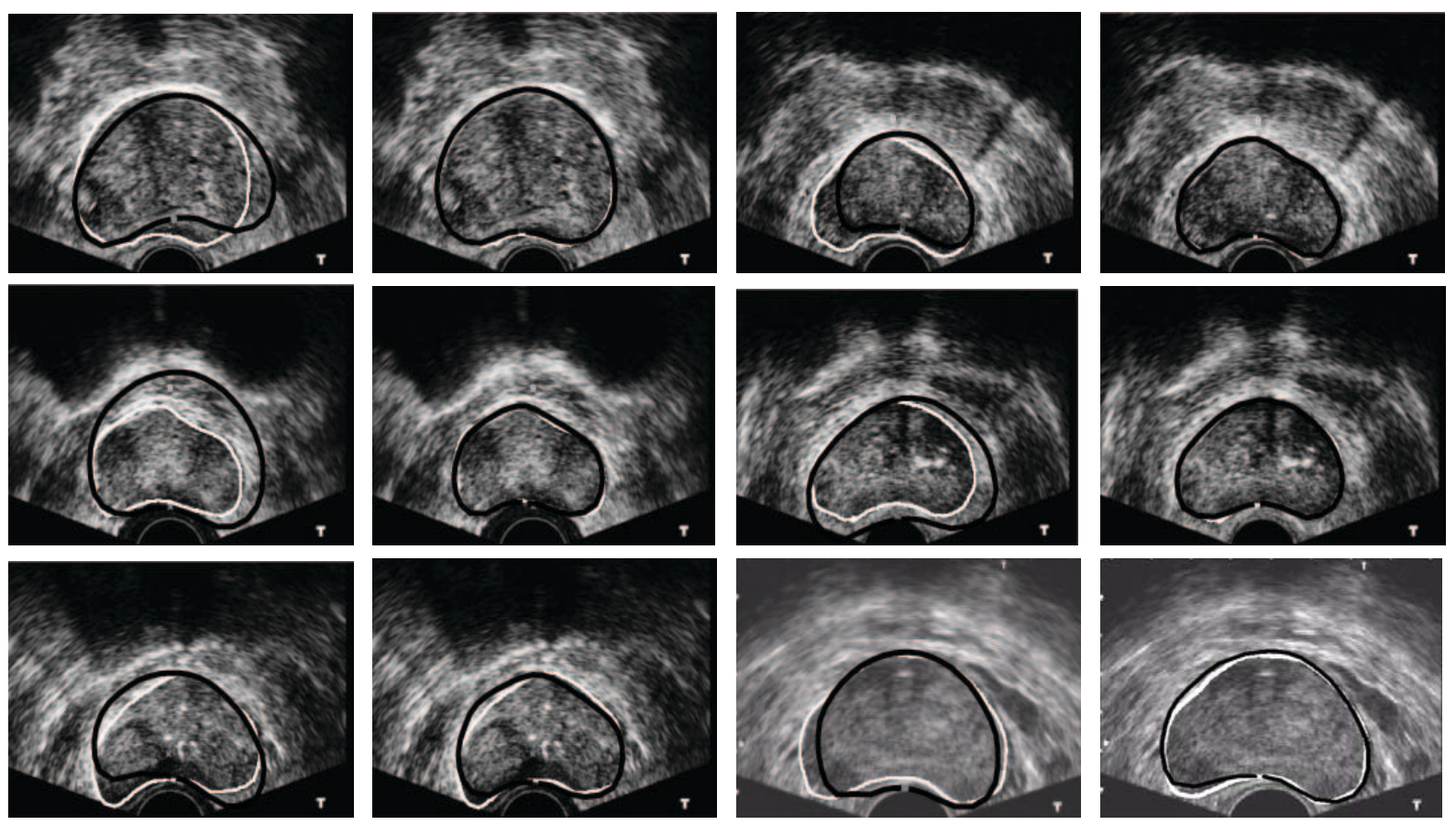

Fig. 3. Performance of our algorithm against shape, size and contrast variations for different patients. The white contour gives the ground truth and the black contour gives the obtained result. Columns 1, and 3 show segmentations with traditional AAM and 2, and 4 show corresponding segmentations with our model.

exists [18]. We plan to exploit the GPU environment with an optimized $\mathrm{C}++$ code to produce close to real time segmentation of the prostate.

\section{CONCLUSION AND FUtURE WORKS}

A novel approach of multiple statistical models of shape and posterior probability information of prostate region with the goal of segmenting the prostate in 2D TRUS images has been proposed. Our approach is accurate, and robust to significant shape, size and contrast variations in TRUS images compared to traditional AAM and some existing work in literature. While the proposed method is validated with prostate mid gland images, effectiveness of the method against base and apical slices will be validated in our future work.

\section{Acknowledgements}

This research has been funded by VALTEC 08-1-0039 of Generalitat de Catalunya, Spain and Conseil Régional de Bourgogne, France.

\section{REFERENCES}

[1] "Prostate Cancer," American Cancer Society Atlanta, GA [Online]. Available: http://www.cancer.org/, accessed on [28th June, 2011], 2011.

[2] P. Yan et al., "Discrete Deformable Model Guided by Partial Active Shape Model for TRUS Image Segmentation," IEEE Trans. on Biomed. Engg., vol. 57, pp. 1158-1166, 2010.

[3] S. Badiei et al., "Prostate Segmentation in 2D Ultrasound Images Using Image Warping and Ellipse Fitting," in MICCAI, 2006, pp. 17-24.

[4] H. M. Ladak et al., "Prostate Segmentation from 2D Ultrasound Images," in Proc. of IEEE EMBS, 2000, pp. 3188-3191.
[5] D. Shen et al., "Segmentation of Prostate Boundaries from Ultrasound Images Using Statistical Shape Model," IEEE Trans. on Medical Imaging, vol. 22, pp. 539-551, 2003.

[6] Y. Zhan and D. Shen, "Deformable Segmentation of 3D Ultrasound Prostate Images Using Statistical Texture Matching Method," IEEE Trans. on Medical Imaging, vol. 25, pp. 256-272, 2006.

[7] F. A. Cosío, "Automatic Initialization of an Active Shape Model of the Prostate," Medical Image Analysis, vol. 12, pp. 469-483, 2008.

[8] T. F. Cootes et al., "The Use of Active Shape Model for Locating Structures in Medical Images," Image and Vision Computing, vol. 12, pp. 355-366, 1994.

[9] T. Cootes et al., "Active Appearance Models," in Proc. of ECCV, Springer, 1998, pp. 484-498.

[10] S. Ghose et al., "Texture Guided Active Appearance Model Propagation for Prostate Segmentation," MICCAI workshop PCI, LNCS, Springer, vol. 6367, pp. 111-120, 2010.

[11] J. C. Gower, "Generalized Procrustes Analysis," Psychometrika, vol. 40, pp. 33-51, 1975.

[12] R. O. Duda et al.,Pattern Classification, 2nd ed. Wiley-Interscience, USA, 2000.

[13] J.Besag, "Spatial Interaction and the Statistical Analysis of Lattice Systems," Jrnl. of Ryl. Statistical Society, vol. 36, pp. 192-236, 1974.

[14] S. Geman and D. Geman, "Stochastic Relaxation, Gibbs Distributions and the Bayesian Restoration of Images," IEEE Trans. on Pattern Analysis and Machine Intelligence, vol. 6, pp. 721-741, 1984.

[15] Y. Boykov, O. Veksler, and R. Zabih, "Fast Approximation Energy Minimization via Graph Cut," IEEE Trans. on Pattern Analysis and Machine Intelligence, vol. 23, pp. 1222-1238, 2001.

[16] C. Rother et al., "Grabcut: Interactive Foreground Extraction using Iterated Graph Cuts," in SIGGRAPH, 2004.

[17] Y. Boykov and M. P. Jolly, "Interactive Graph Cuts for Optimal Boundary and Region Segmentation of Objects in N-D Images," in In Proc. IEEE Int. Conf. on Computer Vision, 2001.

[18] A. Barbu, "Learning Real-Time MRF Inference for Image Denoising," in In Proc. IEEE CVPR, 2001.

[19] MICCAI, "2009 prostate segmentation challenge" http://wiki.namic.org/Wiki/index.php, accessed on [1st April, 2011], 2009. 